\title{
Publisher Correction: Social influence maximization under empirical influence models
}

Sinan Aral (1) and Paramveer S. Dhillon

Correction to: Nature Human Behaviour https://doi.org/10.1038/s41562-018-0346-Z, published online 21 May 2018.

In the version of this Letter originally published, in the key for Fig. 1 the red square was mistakenly labelled 'Low influence' and 'High susceptibility' but should have been labelled 'High influence' and 'Low susceptibility'. This has now been corrected. 